\title{
I. THE ROLE OF RESEARCH ETHICS COMMITTEES IN OBSERVATIONAL STUDIES: EPIDEMIOLOGICAL REGISTRIES, CASE REPORTS, INTERVIEWS, AND RETROSPECTIVE STUDIES
}

\author{
Alejandra González-Duarte*, Martha Kaufer-Horwitz ${ }^{\ddagger}$, Elena Zambrano, Marta Durand-

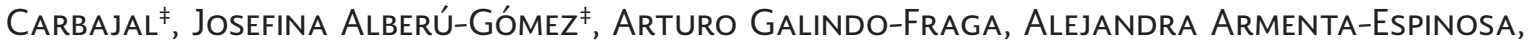 \\ Alvar loria-Acereto ${ }^{\ddagger}$, Marina Rull-Gabayet ${ }^{\ddagger}$, Heriberto Medina-Franco ${ }^{\ddagger}$, Mauricio Sierra-Salazar, \\ Carlos A. Hinojosa ; jorge Oseguera-Moguel, Álvaro Aguayo-González, Patricia Domínguez- \\ Sánchez, Sergio Hernández-Jiménez and Carlos A. Aguilar-Salinas ${ }^{\ddagger}$, for the Ethics Committee \\ and ₹Research Committee, Instituto Nacional de Ciencias Médicas y Nutrición Salvador Zubirán, \\ Mexico City, Mexico.
}

\begin{abstract}
It is often unclear to the clinical investigator whether observational studies should be submitted to a research ethics committee (REC), mostly because, in general, no active or additional interventions are performed. Moreover, obtaining an informed consent under these circumstances may be challenging, either because these are very large epidemiological registries, or the subject may no longer be alive, is too ill to consent, or is impossible to contact after being discharged. Although observational studies do not involve interventions, they entail ethical concerns, including threats such as breaches in confidentiality and autonomy, and respect for basic rights of the research subjects according to the good clinical practices. In this context, in addition to their main function as evaluators from an ethical, methodological, and regulatory point of view, the RECs serve as mediators between the research subjects, looking after their basic rights, and the investigator or institution, safeguarding them from both legal and unethical perils that the investigation could engage, by ensuring that all procedures are performed following the international standards of care for research. The aim of this manuscript is to provide information on each type of study and its risks, along with actions to prevent such risks, and the function of RECs in each type of study. (REV INVEST CLIN. 2019;71:149-56)
\end{abstract}

Key words: Observational studies. Registries. Interviews. Polls. Case reports. Retrospective studies. Informed consent. Research ethics committee.

Corresponding author:

*Alejandra González-Duarte

Departamento de Neurología

Instituto Nacional de Ciencias Médicas

y Nutrición Salvador Zubirán

Vasco de Quiroga, 15

Col. Belisario Domínguez Sección XVI, Del. Tlalpan

C.P. 14080, Mexico City, Mexico

E-mail: gonzalezduarte@aol.com

Received for publication: 06-05-2018

Approved for publication: 09-07-2018

DOI: $10.24875 / R I C .18002580$ 


\section{INTRODUCTION}

Although by definition in an observational study, the investigator is not performing an active procedure, a review from the research ethics committee (REC) is often required. It is less clear if the signature of an informed consent (IC) should be obtained in this context, but it certainly presents a challenge in most occasions, particularly when the study is very large, the patient is too ill to consent, dies soon after hospital admittance, or is difficult to contact.

An "observational study" is defined as a research study based on the information recorded from previous or prospective visits, without additional procedures being done for the sole purpose of the investigation ${ }^{1}$. The term includes retrospective studies such as case and control studies, small or large prospective cohort studies such as epidemiological registries, interviews, case series, case reports, and descriptive studies (Fig. 1).

While questionnaires are, for the most part, not harmful, they are considered interventions when they are not part of routine care. Observational studies are considered to entail none or minimal risk for the subject of the study, according to the current international guidelines ${ }^{1,2}$.

Certainly, observational studies do not confer risk of physical injuries to the study subjects. Thus, clinical researchers may believe that because of their innocuous

Figure 1. Classification of observational studies.

Observational studies can be analytical or descriptive depending on the presence of a comparison group. Cohort studies proceed in a logical sequence from exposure to outcome, whereas case-control studies work backward. Descriptive studies show the frequency, natural history, and possible determinants of a rare condition in case series or case report, whereas cross-sectional studies, which may or may not have a comparison group, examine the presence or absence of disease or exposure simultaneously ${ }^{1}$.

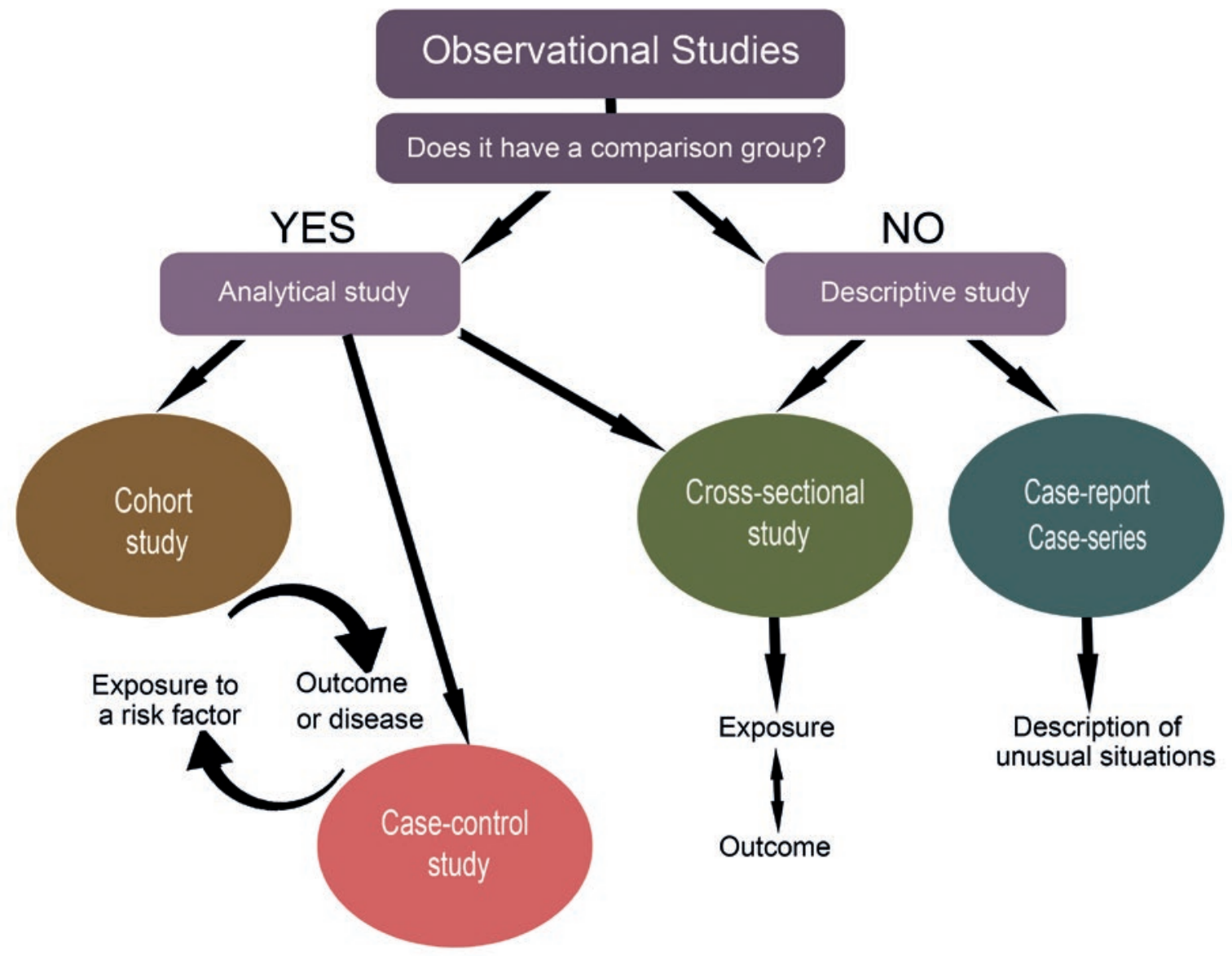


Table 1. Ethical principles in research

\begin{tabular}{ll}
\hline Non-maleficence & $\begin{array}{l}\text { Causing no physical, moral, economic, psychological, or other damages directly related to the } \\
\text { investigation }\end{array}$ \\
Justice & To ensure equal distribution of the subjects of investigation; to avoid exploitation of vulnerable \\
groups only because they are more accessible or submissive \\
To be able to deliberate about personal gears and act accordingly. Three core values linked to \\
autonomy are as follows: \\
Intimacy (spiritual context that makes reference to the most interior and reserved part of each \\
person), privacy (right to determine and control personal information), and confidentiality (right to \\
decide if the private information may be disclosed to third parties) \\
To treat subjects with ethics, respecting not only their decisions but also protecting them from other \\
non-intended purposes and assuring their wellness
\end{tabular}

nature, it is futile to submit the study to an REC review. Moreover, they may also wrongly believe that the information registered in the clinical file is available for them to use to any end, forgetting that the information was recorded for the exclusive purpose of the patient's care. Using information from the clinical file without the patient's authorization violates one of the basic principles of research: the principle of autonomy (for definitions of the ethical principles, Table 1); furthermore, if the researcher is not directly involved in the patient's care, but has access to the medical file, the principle of confidentiality is also breached $^{3}$.

Observational studies are paramount for research. These contribute important information about the natural history of distinct diseases that can be followed up and compared within different populations. Other contributions of these studies are to obviate unnecessary or redundant investigations, thus saving time and resources; evaluate the trend of a particular disease in a certain point in time, and its prognosis, and outcomes of treatments; and eliminate research biases because the collected information is not preanalyzed. They also reveal information that could not have been collected in another type of study because of ethical implications such as an unplanned pregnancy while receiving an investigational agent, exposure in fetuses, or research in children or vulnerable groups, among others.

Reaching a balance between clinical research and patients' rights is paramount (Table 2 ). This can only be achieved when the following two premises are considered: first, that observational studies entail ethical concerns including threats such as breaches in confidentiality and autonomy and respect for the basic rights of research subjects according to the good clinical practices ${ }^{4}$; second, that the efforts to protect the confidentiality of the medical file and the subject of the investigation may endanger clinical research, consume valuable time, and slow the potential benefits of the investigation ${ }^{5}$. All these obstacles are further complicated by each country's different ethical and legal frames of the research procedures ${ }^{6}$. The aim of this manuscript is to review the ethical aspects of observational studies on human subjects and the actions that the REC takes in each type of study.

\section{EPIDEMIOLOGICAL REGISTRIES}

A registry is a document that contains health information of a certain type of subject in any format (paper, electronic, audio, video, etc.). An epidemiologic registry is one created for a specific geographical area, of subjects that present particular diseases, medical conditions, or characteristics, and can be monitored for some time. Some examples are the epidemiologic registries of rare diseases or prospective cohorts to evaluate risk factors from a certain time or population. By definition, these studies are observational, and no further intervention is performed on the study subject.

The epidemiological registry can risk the confidentiality and integrity of its participants. This may have a negative impact on the physical integrity as well as on the social, moral, and employment domains. Moreover, it is possible that the creation of a registry is scientifically and ethically acceptable, but the results are not justified, such as some registries sponsored by pharmaceutical companies, in which the evidence created goes beyond a scientific question. 
Table 2. Actions to avoid conflict in clinical research

\begin{tabular}{|c|c|}
\hline Research ethics committee & Clinical investigator \\
\hline \multirow{9}{*}{$\begin{array}{l}\text { Assesses the research process to protect } \\
\text { both the investigator and the study subject }\end{array}$} & Must describe in detail: \\
\hline & Who will be included in the study \\
\hline & Where will the subjects be found \\
\hline & How will the subjects be communicated with \\
\hline & Who will collect the information \\
\hline & Where will the information be stored \\
\hline & How will the information be protected \\
\hline & Who will have access to the information \\
\hline & How long will the investigator keep the information \\
\hline \multirow{2}{*}{$\begin{array}{l}\text { Evaluates the study pertinence and the } \\
\text { risk-benefit ratio }\end{array}$} & Methods to protect confidentiality \\
\hline & Access codes and passwords \\
\hline $\begin{array}{l}\text { Makes sure that the investigator and the team have } \\
\text { the necessary training and credentials to perform the } \\
\text { investigation, in terms of basic good clinical practice } \\
\text { training }\end{array}$ & $\begin{array}{l}\text { Should specify if additional studies or questionnaires will be } \\
\text { included }\end{array}$ \\
\hline \multicolumn{2}{|l|}{ Follows up the investigation through annual and final reports } \\
\hline $\begin{array}{l}\text { Mediates the ethical breaches that may rise throughout all } \\
\text { the research process }\end{array}$ & \\
\hline
\end{tabular}

It is considered unethical to carry out such studies without the approval from the REC, even if there are no additional procedures performed. Among other things, the REC will ensure that confidentially is respected, will determine if an IC is required, or will grant a waiver for particular circumstances when applicable. This can be performed for all cases that arrive to the institution or as a study protocol. On the other hand, if data are collected from the clinical files, the study should disclose that the information used was not intended for research purposes. To avoid misconduct when creating a registry, researchers should follow ethical principles, such as respecting the dignity of the human subjects involved, and follow these guidelines ${ }^{7}$ :

1. The creation of a registry with aims of investigation should be justified in terms of its scientific merits and social usefulness.

2. The aims and use of the registry should be predefined.

3. If there is a possibility that the rights to the database could be given to someone else, it should be stated from the beginning.
4. The information obtained should be justified in relation to the aims of the study, particularly with sensible information (ethnic groups, religious beliefs, sexual preferences, etc.).

5. There should be a responsible investigator and a public or private institution that collects and protects the information.

6. There should be infrastructure, organization and internal rules, definition of responsibilities, and work procedures.

7. All information relative to the registry must be documented in a protocol to facilitate its correct functioning and evaluation.

8. The protocol should state the objectives of the registry, the need that it pretends to fulfill, and the means to do it. Furthermore, it should also state the information that will be collected, the anonymity process, and the data management.

The most common strategy for the registries is to ask for an IC specifically designed for the study, which 
authenticates the study both ethically and legally. However, this is challenging in large-scale studies. If the subject is unable to participate because of the lack of IC, many studies will be biased. Take, for example, a stroke registry that aims to identify the risk factors within a population where some subjects die before the IC process is carried out. If those subjects are not included in the study, the results will have a selection bias. It may be possible for a proxy or family member to consent on their behalf, but this is not always possible to achieve. Moreover, even if the relative consents to the use of the information, there is no assurance that the subject would have wanted the information to be disclosed. This underscores the difficulty of requiring ICs for participating in an epidemiological registry.

It may be possible to rescind the IC in some registries, but the exception must be justified by the principal investigator for each specific case; this should be evaluated and approved by the REC. A more generalized exception could be considered in studies where the objective is to measure the incidence or prevalence of a certain disease or risk factor, and a massive loss of cases or selection bias will occur if only those that could sign the IC form are included in the study ${ }^{8}$. In the future, alternatives such as electronic ICs or confidentiality agreements could be considered, but there are no regulations approved for this at the moment. To grant an exception to the IC, some fundamental questions should be asked ${ }^{7,9}$ :

a. Is there a real need to perform the study, or do other alternatives exist?

b. How sensible is the information that will be collected?

c. Will knowledge be increased considerably after the study?

d. Are there actions being taken to avoid leaks of information or breaches of confidentiality?

\section{RETROSPECTIVE STUDIES}

In this type of studies, the information that will be used has previously been obtained and registered in a clinical file. Because the subject may or may not be aware that the information is being used for research, confidential personal information can be disclosed. Two different scenarios are possible: when the physician taking direct care of the patients is the investigator, or when the investigator has never met the patients. In the first case, there is no confidentiality violation, as the physician has full access to the information. However, having access to the information does not mean that he or she is capable of using it for any other purpose. The patients assume that the information they disclosed was meant for diagnosis or treatment, not for research. In the second scenario, where an investigator other than the care provider gathers the information, if the patients did not consent to the review of their medical record by another source, a breach of confidentiality may apply to the investigator and to the institution. From an ethical point of view, the principle of autonomy is thus violated, as patients have the right to decide if they want to participate in any type of research. Unfortunately, this principle is violated in most retrospective studies $^{8}$.

RECs may ease these violations if the studies are submitted to reviewing before initiating the study. By allowing that the REC oversees the retrospective studies, the institution and the investigator are being protected because such committees in addition to reviewing the study protocol make sure that researchers are trained to handle sensible information.

Ideally, the patient should specifically consent for the use of his or her information for a different purpose than what it was originally intended. This is not always possible, and the exception is made when the investigation proves to be valuable and information will be used without identifiers. Some institutions have a policy of informing all patients on admission of the possibility of using their information by any member of the institution. Consent forms are given at the time of admission, and the patient has the option to deny or grant his or her information or to ask to be contacted every time to learn about the specific study. If the patients deny the use of their information or ask for more information about the study, there should be no consequences, and medical care must continue as it was originally planned, not affecting the patient. It is paramount that such consent forms are reviewed by an REC and ensure good clinical practice policies. 
Finally, when it is impossible to obtain the IC, the REC may waive its use in the following circumstances ${ }^{10,11}$ :

a. The interest of the study exceeds the personal interests of the patients.

b. It would be almost impossible to obtain IC from the patient.

c. The investigator has the commitment to maintain the confidentiality for the patient.

\section{INTERVIEWS AND POLLS}

Valuable information can be collected from direct interviews, particularly when the study subject has a low education profile or does not speak or understand the language fully. Interviews can improve the relationship between the participant and the interviewer and disclose useful information that is difficult to acquire by other means. However, the impact that this proximity may have on some particularly with certain topics (e.g., traumatic experiences and sexual practices, among others). The researcher may unintentionally harm the patient by the content of the questions, the way the questions are formulated, the facilities where the interviews take place, or persons present at the time of the interview. According to the World Medical Organization ${ }^{4}$, the psychological or social harm of the interviews may include:

a. Discussion of traumatic experiences or private issues that the subject does not wish to remember.

b. Violation of confidentiality and privacy.

c. Fear of stigmatization by the subject when disclosing private information.

d. Feeling of vulnerability.

In general, interviews are considered research with "minimal or less than minimal risk" for the participant $^{2}$. Therefore, they could be reviewed in an expedite fashion by the REC given that all the information gathered will remain anonymous. Even with written questionnaires, the investigator should make sure that there is an appropriate time and place to perform the interview, where the subject will feel comfortable to disclose the information.
The subject should receive a written document which does not substitute the verbal explanation that must expose, with very simple language, all the relevant information: objectives of the interview, actions which will be taken to protect the participant's confidentiality, number of visits, estimated time of completion, expenses, any other difficulties which the subject may confront, who will have access to the information, and expose clearly that the subjects' partaking is voluntary, and his or her refusing to participate will not carry any consequences to their clinical care.

\section{CASE REPORTS}

Case reports are the type of research most likely to endanger confidentiality because sometimes it may be difficult to disguise the subject's information. Twelve editors from different journals gathered in 1995 and reached a consensus regarding case reports. The following measures were suggested ${ }^{12}$ :

a. Patients have a right for privacy that should not be violated; therefore, the IC process and a written IC form are always necessary.

b. The case report should avoid information that could be recognized, such as detailed explanations, photographs, or pedigrees, unless it is absolutely relevant.

c. Because anonymity is difficult to obtain in these cases, the patients should review the final manuscript and additional files before consenting to participate.

Case reports do not require an approval from the REC, except in some circumstances, for example, if the study is meant to be a graduation work such as a thesis, or if it contains sensible information or photographs. However, they do need a signed IC. The IC will bring protection to the subject, to the investigator, and to the journal where the case is meant to be published. In cases in which a written IC cannot be obtained and personal information may be revealed, the case should not be published because it is preferable to protect the patient's privacy than the knowledge that the case could bring. It is not justified to alter information with the sole purpose of protecting confidentiality. It is still uncertain if it is acceptable to publish a case report on 
Table 3. Requirements for each type of observational studies

\begin{tabular}{lcc}
\hline Type of study & REC review & Written informed consent \\
\hline Epidemiological registry & Yes & Yes** $^{* *}$ \\
Retrospective study & Yes* & Yes** $^{* *}$ \\
Case Report & No (studies for obtaining & Yes** \\
Interviews and Polls & a professional degree may need it) & Yes \\
\hline
\end{tabular}

*May apply for an expedite review when the study does not exceed more than the minimal risks (Table 4 ). ${ }^{* *}$ May apply for a waiver when the study does not exceed more than the minimal risks (Table 4).

Table 4. More than minimal risks in observational studies*

\begin{tabular}{|c|c|c|}
\hline Action & Definition & Exception \\
\hline Departure from normal care & $\begin{array}{l}\text { Something done or withheld from } \\
\text { a patient that deviates from } \\
\text { normal health care (i.e., when } \\
\text { extra blood samples are taken) }\end{array}$ & \\
\hline Use of stored samples & $\begin{array}{l}\text { Use, collect, or store human } \\
\text { samples without IC or for } \\
\text { purposes other than those for } \\
\text { which they were originally } \\
\text { collected }\end{array}$ & $\begin{array}{l}\text { Participants gave IC to future unspecified use } \\
\text { If a statutory exception to the need to gain consent } \\
\text { applies } \\
\text { To ensure quality of services: } \\
\text { Rereading specimens to check for accuracy } \\
\text { Audits } \\
\text { Evaluation of services }\end{array}$ \\
\hline $\begin{array}{l}\text { Use of identifiable information } \\
\text { collected for a secondary } \\
\text { purpose }\end{array}$ & $\begin{array}{l}\text { Confidential sensitive information } \\
\text { collected for clinical care used } \\
\text { for research }\end{array}$ & $\begin{array}{l}\text { Statutory exception to the need to gain informed } \\
\text { consent }\end{array}$ \\
\hline & & Purpose of quality assurance \\
\hline
\end{tabular}

*Modified from ethical guidelines for observational studies: Observational research, audits, and related activities ${ }^{13}$

a patient who died without giving IC. Some local regulations allow its publication when all identifiers are withdrawn, and the case will show relevant new information, but this varies between countries.

\section{CONCLUSIONS}

Proactive clinical investigators will foresee the possible complications and ethical concerns of their research and will be more successful in their investigations and more principled than those less vigilant. When contemplating the risks and research misconducts before and during an investigation, arrangements may be taken to prevent conflicts related to an action that is well intended but may elicit legal or psychological conflicts if it is not performed properly
(Table 3). The aim of the REC is to evaluate research protocols from the ethical, methodological, and regulatory point of view. However, it serves both interests, the investigators', and the study subjects', by overseeing that the research is performed following the current regulations. To evaluate a research protocol, RECs use the basic principles of ethics in research but, also, grant the possibility of considering that they are not absolute and recognize that each case presents its own challenges. Hence, to reach a valid conclusion, a careful multidisciplinary review of each protocol individually is mandatory, even in observational studies.

Acknowledgments: We would like to thank Arch. Héctor Zambrano González for the edition of figure 1, and Dr. Sergio Iván Valdés-Ferrer for reviewing the final version of the manuscript. 


\section{REFERENCES}

1. Grimes DA, Schulz KF. An overview of clinical research: the lay of the land. Lancet. 2002;359:57-61.

2. Council for International Organizations of Medical Sciences $(\mathrm{Cl}-$ OMS) in Collaboration with the World Health Organization. International Ethical Guidelines for Epidemiological Studies. Geneva: Council for International Organizations of Medical Sciences $(\mathrm{Cl}$ OMS) in Collaboration with the World Health Organization; 2009.

3. Masic I, Hodzic A, Mulic S. Ethics in medical research and publication. Int J Prev Med. 2014;5:1073-82.

4. World Medical Association. Medical Ethics Manual. 3rd ed. Ch. V. Medical Research. Manual for physicians about the role of ethics in medicine. World Medical Association; 2015.

5. Perlam M. Ethical issues in observational research. Paedriat Child Health. 2000;5:89-90.

6. Ludvigsson J, Haberg S, Knudsen $\mathrm{P}$, et al. Ethical aspects of a registry-based research in the Nordic countries. Clin Edipemiol. 2015;7:491-508.
7. Medical Research Council "Personal Information in Medical Research". Available from: http://www.mrc.ac.uk/Utilities/Documentre-cord/index.htm?d=MRC002452.

8. Drazen J, Harrinton D, McMurray J, et al. The challenging face of clinical trials. N Engl J Med. 2016;375:454-63.

9. De abajo Iglesias F, Feito Grande L, Júdez Gutierrez J, et al. Directrices éticas sobre la creación y usos de registros con fines de investigación biométidica. Rev Esp Salud Publica 2008; 82:21-42.

10. Melton LJ. III The threat to medical-records research. N Engl ] Med. 1997;337:1466-70.

11. Junod V. Retrospective research: what are the ethical and legal requirements? Swiss Med Wkly. 2010;25:140.

12. Levine S, Stagno S. Informed consent for case reports. J Phsycother Pract Res. 2001;10:193-201.

13. National Ethics Advisory Committee. Ethical Guidelines for Observational Studies: Observational Research, Audits and Related Activities. Revised Edition. Wellington, NZ: Ministry of Health 2012. Available from: http://www.neac.health.govt.nz. 